\title{
Review of: "Medicines for the new coronavirus in the view of Classical Systemic Homeopathy"
}

\author{
Robbert van Haselen, Yvonne Fok, Alexander Tournier ${ }^{1}$
}

1 University of Berne

Potential competing interests: The author(s) declared that no potential competing interests exist.

We have read the article on homeopathic medicines for the new coronavirus with interest.

As the authors point out, homeopathy has a rich history of use in epidemics. The COVID-19 pandemic has led to various initiatives to further explore the possible role of homeopathy. Much work is still needed in this area and the authors' contribution in this area is welcome. We would like to share some comments, as well as our own observations, which we feel will enrich the discussion around this topic.

Firstly, as a minor point, the authors refer to the now obsolete term 'genius epidemicus', which was regarded by the ancients as the purported cause of epidemic diseases. The common elements of the symptomatic picture that can be observed during epidemics, as well as its corresponding homeopathic remedy, are referred to in the homeopathic literature as 'genus epidemicus'.

More importantly, the authors performed a repertorisation of the COVID-19 symptoms reported in the literature, and propose the homeopathic medicine 'Cinchona officinalis' as the most suitable genus epidemicus remedy. It is our opinion that symptoms selected from the published literature may lack sufficient specificity from a homeopathic perspective, and we therefore wonder why the authors did not choose to also include data from actual cases.

The Clificol COVID-19 support project is the largest homeopathic COVID-19 case registry worldwide. It is an online multi-national, cloud-based clinical case registry that is supported by all the major professional associations. At the time of our analysis (11-11-21), the database contained 1174 COVID-19 cases, which received in total 1628 different homeopathic prescriptions. Only $17(1 \%)$ of all these prescriptions concerned Cinchona officinalis. Using the ORIDL ${ }^{1}$ outcome assessment tool we found that 10 of these continued with the remedy and concluded as 'back to normal' either without (7) or with (3) sequelae. 3 cases were not yet concluded, and in 4 cases a change of remedy was needed due to lack of effectiveness (3) or due to the need for a follow-up remedy (1). In 7 of the 17 Cinchona prescriptions, no rubrics were entered. In the 10 Cinchona cases where rubrics were recorded, fever related symptoms were most common, including the classical symptom 'fever, alternating with chills' was recorded for two prescriptions. On the other hand, chest and respiratory system related symptoms as referred to prominently by Gosik et 
al, were only recorded in 4 prescriptions.

We have recently completed a genus epidemicus analysis of the Chinese population which gives strong indications that there were two symptomatic presentations, corresponding with the Gelsemium (78\% of cases) and Bryonia (18\% of cases) remedy pictures. Modern statistical analysis tools clearly showed that these two groups are well defined and distinct. Consequently, averaging over all rubrics in order to obtain the Genus Epidemicus makes little sense.

We are currently analysing the data from the rest of the world. Preliminary analysis showed that other remedies such as Phosphorus and Arsenicum were also highly prescribed. Overall, these analyses indicate that Cinchona is neither a common, nor a highly effective remedy.

Whilst analysis of symptoms reported in the literature can be useful, it is important to obtain 'real-world' data from clinical practice for more definitive answers. Our analyses also indicate that a narrow focus on a single genus epidemicus remedy is not justified and that COVID-19 is associated with at least 2 genus epidemicus remedies.

\section{References}

1. Reilly D, Mercer SW, Bikker AP, Harrison T. Outcome related to impact on daily living: Preliminary validation of the ORIDL instrument. Article. BMC Health Services Research. 2007;7139. doi:10.1186/14726963-7-139 